OPEN ACCESS

Edited by:

Leonard Peruski,

US Centers for Disease Control and Prevention, USA

Reviewed by:

Haider Abdul-Lateef Mousa,

University of Basrah, Iraq

Yunlong Li,

Wadsworth center, USA

${ }^{*}$ Correspondence:

Tuanyuan Shi

Istone2008@126.com;

Xun Suo

suoxun@cau.edu.cn

tThese authors have contributed equally to this work

Specialty section:

This article was submitted to Infectious Diseases,

a section of the journal

Frontiers in Microbiology

Received: 24 December 2015 Accepted: 12 May 2016

Published: 31 May 2016

Citation:

Shi T, Tao G, Bao G, Suo J, Hao L,

$F u Y$ and Suo $X(2016)$ Stable Transfection of Eimeria intestinalis and Investigation of Its Life Cycle, Reproduction and Immunogenicity.

Front. Microbiol. 7:807.

doi: 10.3389/fmicb.2016.00807

\section{Stable Transfection of Eimeria intestinalis and Investigation of Its Life Cycle, Reproduction and Immunogenicity}

\author{
Tuanyuan Shi ${ }^{\text {*t}}$, Geru Tao ${ }^{2 t}$, Guolian Bao ${ }^{1}$, Jingxia Suo ${ }^{2}$, Lili Hao ${ }^{3}$, Yuan $\mathrm{Fu}^{1}$ and \\ Xun Suo ${ }^{2 *}$ \\ ${ }^{1}$ Department of Animal Parasitology, Institute of Animal Husbandry and Veterinary Medicine, Zhejiang Academy of \\ Agricultural Science, Hangzhou, China, ${ }^{2}$ National Animal Protozoa Laboratory, College of Veterinary Medicine, China \\ Agricultural University, Beijing, China, ${ }^{3}$ College of Life Science and Technology, Southwest University for Nationalities, \\ Chengdu, China
}

Rabbit coccidiosis, caused by infection of Eimeria spp. is one of the most severe parasitic diseases in rabbits. Eimeria intestinalis is one of the most immunogenic species in rabbit coccidia. Due to the lack of genomic information and unsuccessful in vitro cultivation, genetic manipulation of rabbit coccidia lagged behind other apicomplexan parasites. Using regulatory sequences from $E$. tenella, we obtained a transgenic line of $E$. intestinalis expressing yellow fluorescent protein (YFP). YFP was continuously expressed throughout the whole life cycle. Morphological features of $E$. intestinalis in different developmental stages were dynamically observed with the transgenic line. Some important features in the endogenous development stages were observed. Trophozoites were found as early as $4 \mathrm{~h}$ post inoculation. Two types of schizonts and merozoites were observed in first three of the four schizogonies. Beside jejunum and ileum, gametogony stage and oocysts were also found in the duodenum and vermiform appendix. In addition, the transgenic strain was highly immunogenic but less pathogenic than the wild type. Considering the high immunogenicity of $E$. intestinalis and amenability to transfection with foreign genes, transgenic E. intestinalis could be a promising oral eukaryotic vaccine vector.

Keywords: Eimeria intestinalis, YFP, stable transfection, life cycle, reproduction, pathogenicity

\section{INTRODUCTION}

Coccidiosis is one of the most common and highly contagious diseases in rabbits, causing severe economic losses in rabbitries due to reduced weight gain and high mortality. Currently, treatment with anticoccidial drugs is the only effective measure in control of rabbit coccidiosis (Pakandl, 2009). However, vaccination with live oocysts is the preferred method since the use of anticoccidial drugs is associated with the emergence of drug resistance strains and drug residues in rabbit meat products.

Genetic manipulation is a powerful technique to study parasite biology and immunology, and to develop novel vaccines against infectious diseases. Transfection of apicomplexan parasites has transformed the studies of this important group of pathogens. By disruption of the locus or 
replacement with an altered or tagged gene, functions of specific genes have been studied for the apicomplexan parasites Toxoplasma gondii and Plasmodium spp. (Goodewardene et al., 1993; Donald and Roos, 1993; Soldati and Boothroyd, 1993; Sibley et al., 1994; de Koning-Ward et al., 2000; Li et al., 2015a). However, research advances on transfection of Eimeria, which are also of the apicomplexan family, are slower than those of T. gondii and Plasmodium spp. This is mainly because Eimeria spp. cannot be continuously cultivated in vitro. Stable transfection of Eimeria requires preparation and purification of sporozoites from oocysts, inoculation of transfected sporozoites into parasitic sites of host animals and propagation of selected oocysts. Fortunately, stable transfection systems have been successfully established in a rat Eimerian species, Eimeria nieschulzi (Kurth and Entzeroth, 2009), and two chicken Eimerian species, E. tenella (Yan et al., 2009) and E. mitis (Qin et al., 2014).

Eimerian parasites can stimulate strong antibody and cellmediated immunity (Wallach, 2010). Their exceptional potential as vectors delivering heterologous antigens to the mucosal immune system were well recognized (Shi et al., 2008; Yan et al., 2009; Clark et al., 2012). Two transgenic lines of E. tenella were reported to induce exogenous antigen-specific immune responses (Huang et al., 2011; Clark et al., 2012), and another transgenic E. mitis expressing chicken interleukin 2 strongly enhanced cellular immunity against wild type parasite challenge (Li et al., 2015b). However, little information was available on the transfection of rabbit Eimerian species.

In this study, we, for the first time, established a stable transfection system in a rabbit coccidian species, E. intestinalis, which is one of the most immunogenic coccidia species (Licois et al., 1990; Coudert et al., 1993, 1995). Taking advantages of the fluorescence, we identified important features in the endogenous development stages of this parasite. Moreover, this transgenic line showed reduced pathogenicity but maintained high immunogenicity, indicating it could be developed as an antigen delivery vector.

\section{MATERIALS AND METHODS}

\section{Parasites, Animals, and Cell Culture}

A low-virulent E. intestinalis strain, which was isolated and identified by Shi et al. (2014) was used in the study. About $5 \times 10^{7}$ freshly sporulated oocysts were first ground to release sporocysts. Then sporocysts were excysted in a digestion solution (PBS pH7.4, containing 10\% chicken bile and $0.75 \%$ trypsin). Released sporozoites were purified by a DE-52 cellulose column.

Coccidia-free rabbits were reared in a coccidia-free environment in our institute according to the modified therapeutic method described by Coudert et al. (1995). The young rabbits were weaned at the age of 18 days, and then fed human infant formula (Beingmate, China) in combination with rabbit pellets (prepared in our institute) till 30 days old. Feed and water were sterilized and provided ad libitum. Feces was checked daily for any coccidian oocysts.
Primary rabbit small intestine cells (PRICs) were obtained from newborn rabbits and used for transient transfection of E. intestinalis. Newborn rabbit pups prior to their first suckling were euthanized and then immersed in $75 \%$ ethanol for $10 \mathrm{~min}$ to kill external microorganisms. The small intestine including distal duodenum, jejunum and ileum were excised and longitudinally opened, and intestinal contents were removed. The intestinal wall of $0.5 \mathrm{~cm}^{2}$ was washed with sterilized PBS and then digested in collagenase IV solution at $37^{\circ} \mathrm{C}$ for $30 \mathrm{~min}$. PRICs were isolated by centrifugation at $1000 \mathrm{rpm}$ for $5 \mathrm{~min}$, and grown in the RPMI1640 culture medium supplemented with fetal bovine serum $[10 \%(\mathrm{v} / \mathrm{v})]$, penicillin $(200 \mathrm{U} / \mathrm{ml})$, and streptomycin $(20 \mathrm{mg} / \mathrm{ml})$ at $39.5^{\circ} \mathrm{C}$ in an atmosphere of $5 \% \mathrm{CO}_{2}$.

Our research with animals was approved by the Beijing Administration Committee of Laboratory Animals and performed in accordance with the China Agricultural University Institutional Animal Care and Use Committee guidelines.

\section{Plasmid and Parasite Transfection}

The plasmid pEtHEA, constructed in Professor Xun Suo's laboratory, was used to transfect E. intestinalis (Yan et al., 2009). The plasmid carries a $1090 \mathrm{bp}$ promoter from the $5^{\prime}$ flanking sequence of $E$. tenella histone 4 gene (H4), a 1467 bp sequence of tandem yellow fluorescent protein (YFP) gene (YFP-YFP), and a $1402 \mathrm{bp}$ terminator from the $3^{\prime}$ untranslated region sequence of E. tenella actin gene (Actin). Transfection of E. intestinalis sporozoites with $p$ EtHEA was carried out according to the method of Liu et al. (2008). Electroporation was performed using a Gene Pulser X Cell ${ }^{\mathrm{TM}}$ (Bio-Rad, USA) at $2000 \mathrm{v}$ and $25 \mu \mathrm{F}$ with a pulse time of about $0.3 \mathrm{~ms}$. After electroporation, the parasites were left undisturbed at room temperature for at least $20 \mathrm{~min}$, and then transferred to a confluent monolayer of PRICs. Transfected sporozoites were examined under a fluorescent microscope (Olympus, Japan) 24 h post inoculation (pi).

For stable transfection, approximately $1 \times 10^{6}$ transfected sporozoites were surgically inoculated into the duodenum of a
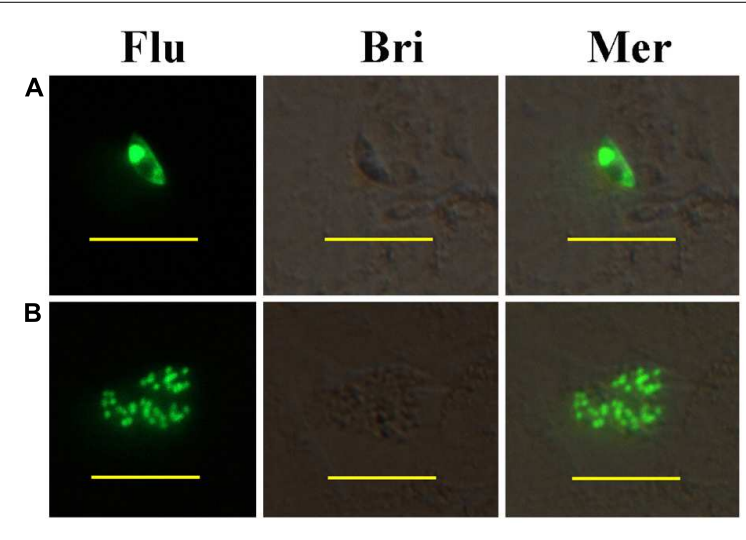

FIGURE 1 | Development of transiently transfected Eimeria intestinalis sporozoites in primary rabbit intestinal cells (PRICs). (A) A sporozoite expressing YFP in PRICs; (B) a first generation schizont expressing YFP in PRICs. Flu, Bri, and Mer indicate fluorescent field, bright field, and merged images, respectively. Bar $=20 \mu \mathrm{m}$. 

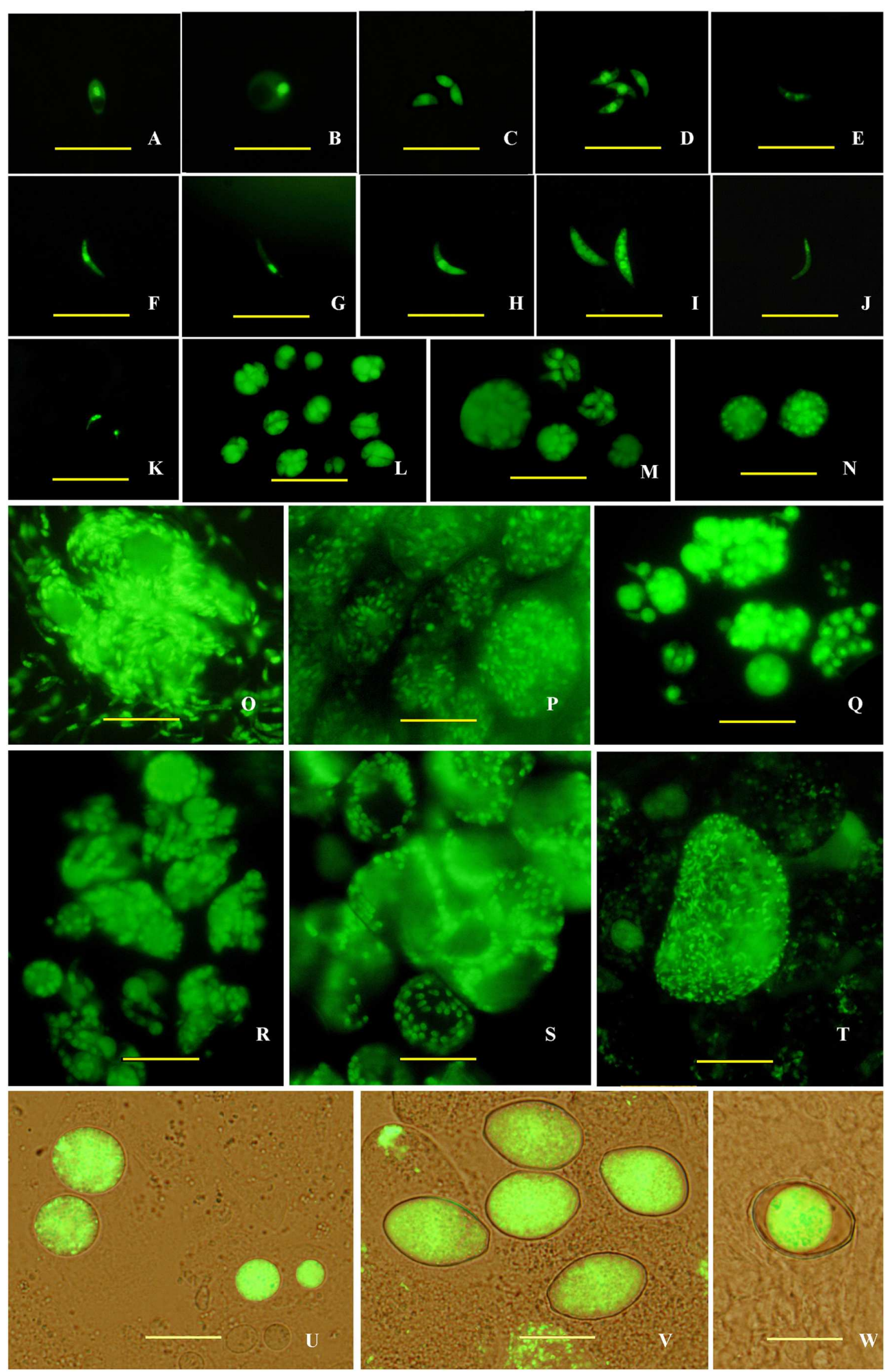

FIGURE 2 | Endogenous development of the transgenic E. intestinalis expressing YFP in rabbits. (A) A sporozoite at $4 \mathrm{~h}$ pi; (B) a trophozoite at $4 \mathrm{~h}$ pi; (C,D) first generation merozoites at $96 \mathrm{~h} \mathrm{pi}$ (E-G) second generation merozoites at $120 \mathrm{~h}$ pi; (H,I) third generation merozoites at $168 \mathrm{~h}$ pi; (J) a fourth generation

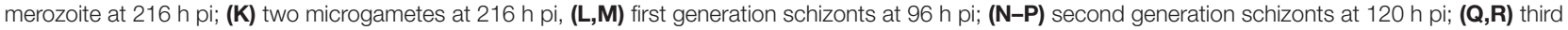
generation schizonts at $168 \mathrm{~h} \mathrm{pi}$ (S) fourth generation schizonts at $216 \mathrm{~h} \mathrm{pi;}$ (T) a microgamont at $216 \mathrm{~h} \mathrm{pi;}$ (U) macrogamonts at $216 \mathrm{~h}$ pi; (V) Zygotes at $216 \mathrm{~h} \mathrm{pi}$; (W) a mature oocyst at $216 \mathrm{~h} \mathrm{pi.} \mathrm{Bar}=20 \mu \mathrm{m}$. 
coccidia-free rabbit. Oocysts from feces excreted between 9 and 14 days pi were collected using the saturated $\mathrm{NaCl}$ flotation method and sporulated in $2.5 \%$ potassium dichromate at $28^{\circ} \mathrm{C}$.

Sporulated oocysts were purified with $13 \%$ sodium hypochlorite solution and then washed with sterilized PBS. Transgenic oocysts were screened by fluorescence- activated cell sorting with a MoFlo Cell Sorter (Dako Cytomation, Fort Collins, CO, USA). Sorted fluorescent oocysts were propagated in coccidia-free rabbits. Stably transfected E. intestinalis were obtained after five cycles of sorting and propagation in coccidia-free rabbits.

\section{Observation of the Life Cycle of Transgenic E. intestinalis}

To study the life cycle of the transgenic E. intestinalis, five rabbits were inoculated with $1 \times 10^{6}$ sporulated oocysts each for observation of early endogenous development stages and four rabbits were inoculated with $1 \times 10^{3}$ sporulated oocysts each for observation of late endogenous developmental stages. Intestine smears were made by scraping the mucosal membrane of duodenum, jejunum, ileum, cecum, vermiform appendix, and colon of the rabbits euthanized 4, 24, 48, 72, 96, 120, 168, 216, and $336 \mathrm{~h}$ pi. For observation of sporogony of the parasites, freshly excreted oocysts were collected at $336 \mathrm{~h}$ pi from fecal samples, and allowed to sporulate in $2.5 \%$ potassium dichromate solution. Observation of the parasites was conducted under a fluorescence microscope.

\section{Determination of Reproduction and Immunogenicity of the Transgenic Parasites}

Sixteen 34-day-old coccidia-free rabbits housed 2 per cage were distributed into four groups with four rabbits per group. One group was orally inoculated with $5 \times 10^{3}$ sporulated oocysts of E. intestinalis wild type. Same amount of oocysts of E. intestinalis transgenic strain were inoculated to another group. Both groups and a non-immunized, challenged control group were orally challenged with $1 \times 10^{6}$ sporulated oocysts of the wild type parasites 16 days pi. The last group was not immunized or challenged and served as a negative control.

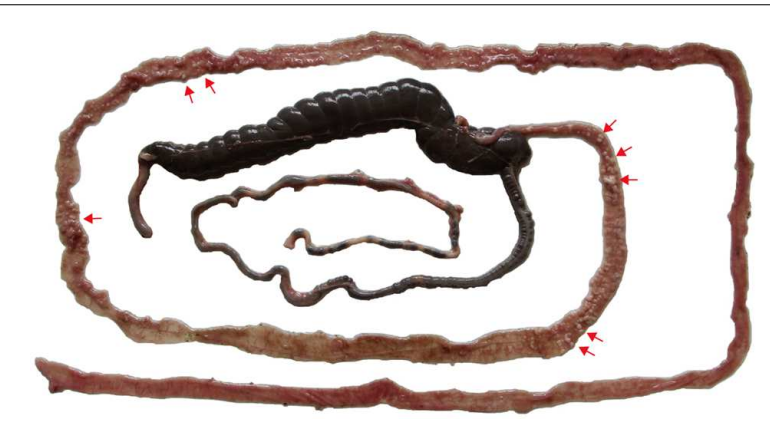

FIGURE 3 | Pathological changes of white nodules (red arrows) of the lower jejunum and ileum of a rabbit at 9 days after inoculation with $5 \times 10^{3}$ transgenic $E$. intestinalis oocysts.
Body weights of all rabbits were measured at 0 and 16 days after immunization and at 10 and 19 days after challenge. Feces were collected daily from 9 to 15 days after immunization and 9 to 16 days after challenge. Total oocyst output in feces were counted in a McMaster chamber.

\section{Statistical Analysis}

Statistical analysis was performed by one-way ANOVA using the SPSS 16.0 software. Data were expressed as mean \pm standard deviation. Differences between groups were considered statistically significant when $p$-values were less than 0.05 .

\section{RESULTS}

\section{Transient Expression of YFP in E. intestinalis}

In vitro, fluorescent sporozoites and first generation schizonts were observed after inoculation to PRICs. YFP was mainly located within nucleus of the parasites (Figures 1A,B). No fluorescence was observed in the refractile body or parasitophorous vacuole. This localization was determined by a nucleus-targeting signal sequence incorporated in the histone 4 promoter of $p$ EtHEA.

\section{Stable Transfected E. intestinalis and Its Development in the Whole Life Cycle}

First, we obtained a stable transgenic line of E. intestinalis. Fluorescent oocysts were observed from rabbit feces on the 9th day after injection of transfected sporozoites. Microscopic observation and cytometry analysis revealed that only $0.01 \%$ of the first generation expressed YFP (data not shown). However, in subsequent passages, fluorescent rate increased to $21.64 \%$ in the second generation and $80 \%$ in the third generation. Similar results with the same plasmid had been reported in E. tenella by Yan et al. (2009), where the percentage of fluorescent oocysts was only $0.04 \%$ in the first generation and rose to $87.5 \%$ in the third generation. Collectively the findings suggested that the constructed sequence of $p$ EtHEA is conserved among the genus Eimeria and it is highly functional and efficient in E. intestinalis.

Then, endogenous development stages of transgenic E. intestinalis in rabbit intestines were observed under a fluorescent microscope between 4 and $216 \mathrm{~h}$ pi. Sporozoites mainly parasitized in the lower jejunum and ileum (Figure 2A), and developed into trophozoites as early as $4 \mathrm{~h}$ pi. These trophozoites were mononuclear and appeared spherical (Figure 2B). They developed into first generation schizonts containing several, usually less than five merozoites (Figures 2L,M) at $72 \mathrm{~h}$ pi. The first generation merozoites were the smallest in four schizogony stages and released at $96 \mathrm{~h}$ pi. Two forms of merozoites were observed: one appeared ovoid (Figure 2C), and the other was boat-shaped with two pointy ends and were slightly longer than the ovoid merozoites (Figure 2D). They all contained one large nucleus located in the middle region of the parasite. Large second generation schizonts containing dozens to hundreds of merozoites (Figures $2 \mathrm{~N}-\mathbf{P}$ ) were found 


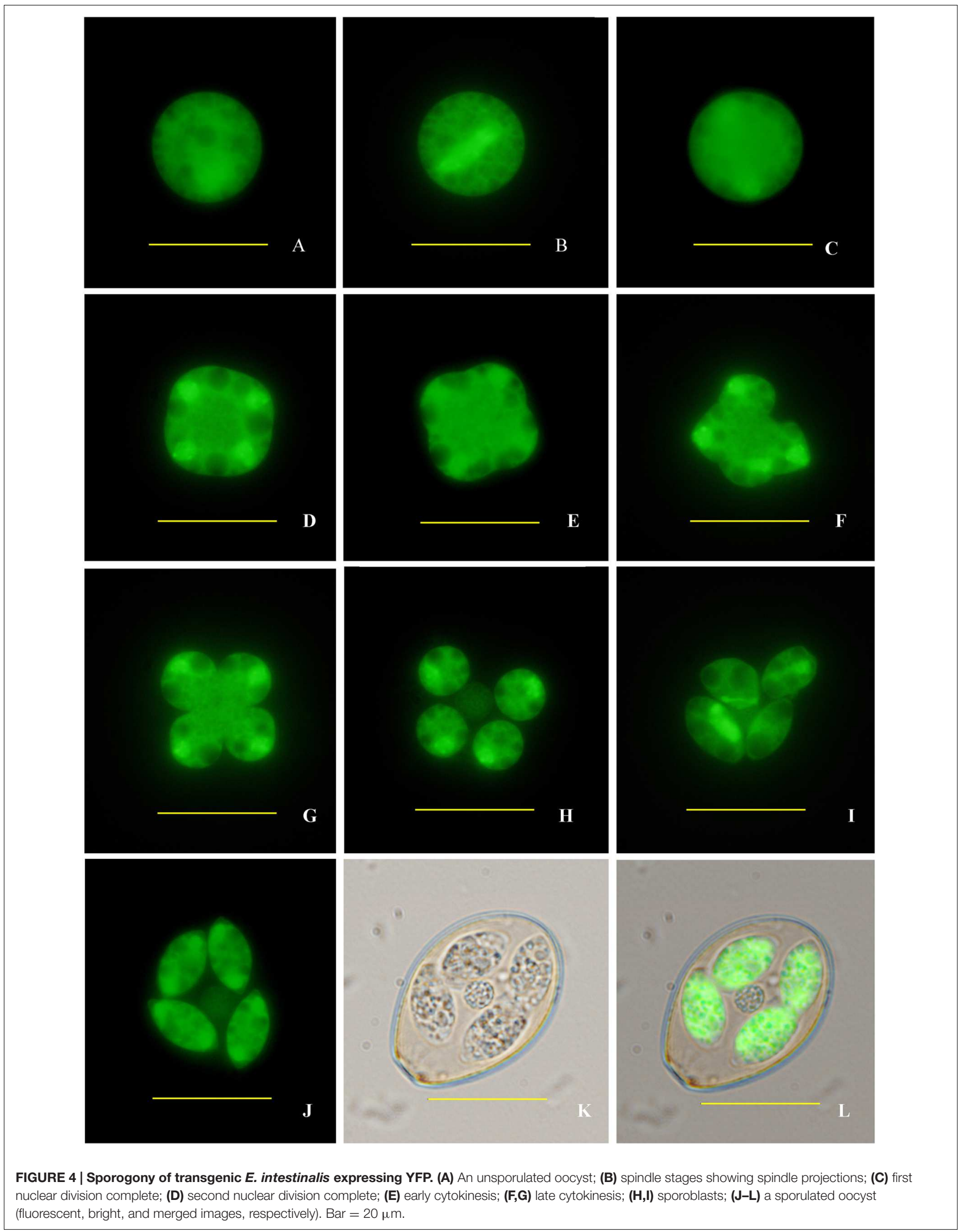


TABLE 1 | Oocyst output in rabbits (mean \pm SD; $n=4)$ after immunization with sporulated wild type or transgenic oocysts $\left(5 \times 10^{3}\right)$ and challenged with the wild type oocysts $\left(1 \times 10^{6}\right)$ of Eimeria intestinalis.

\begin{tabular}{|c|c|c|c|c|}
\hline Immunization & Challenge & $\begin{array}{l}\text { Oocyst output per rabbit } \\
\text { after immunization }\end{array}$ & $\begin{array}{l}\text { Oocyst output per rabbit } \\
\text { after challenge }\end{array}$ & $\begin{array}{l}\text { Reduced percentage of } \\
\text { oocyst output with the } \\
\text { challenge control group }\end{array}$ \\
\hline Wild type oocysts & Wild type oocysts & $19.22 \times 10^{8} \pm 2.069 \times 10^{8}$ & $2.81 \times 10^{8} \pm 0.235 \times 10^{8}$ & $84.35 \%$ \\
\hline Transgenic oocysts & Wild type oocysts & $2.57 \times 10^{8} \pm 0.333 \times 10^{8}$ & $6.79 \times 10^{8} \pm 0.356 \times 10^{8}$ & $62.25 \%$ \\
\hline None & Wild type oocysts & 0 & $18.09 \times 10^{8} \pm 1.815 \times 10^{8}$ & - \\
\hline None & None & 0 & 0 & - \\
\hline
\end{tabular}

at $120 \mathrm{~h}$ pi. The second generation merozoites were also of two types. One was multinuclear and crescent-shaped (Figure 2E), and the other type was uninucleate, banana-shaped and longer than the multinuclear merozoites (Figures 2F,G). Both types of second generation merozoites were more slender than first generation merozoites. The third generation schizonts were observed at $168 \mathrm{~h} \mathrm{pi}$, housed several to dozens of merozoites (Figures 2Q,R) and were smaller than the second generation schizonts. Both two types of the third generation merozoites were the largest of the four schizogony stages. The uninucleate merozoites (Figure $\mathbf{2 H}$ ) was smaller than multinuclear types (usually 4 to 5 nuclei; Figure 2I). There was only one form of Fourth generation schizonts, which contained dozens of uninucleate merozoites and were found at $216 \mathrm{~h}$ pi. The nucleus was located in the anterior end of the merozoites (Figure 2J). A large vesicle was observed in the meronts (Figure 2S).

Parasites in the gametogony stage (including macrogametes, microgamonts, microgametes, and zygotes) and oocysts were mainly found in the lower jejunum and ileum at $216 \mathrm{~h} \mathrm{pi}$, but also found in duodenum and vermiform appendix. Microgamonts contained thousands of microgametes (Figure 2T), which were tadpole- shaped with one nucleus located in the anterior end (Figure 2K). Macrogametes appeared spherical or elliptical (Figure 2U). Zygotes and oocysts all appeared piriform (Figure 2V), but the oocyst wall was obvious in mature oocysts and the cytoplasm was usually condensed into a sphere (Figure 2W). In addition, nodule formation and small intestine wall thickening were found in the lower jejunum and ileum after 9 days pi (Figure 3 ).

Finally, sporogony of transgenic oocysts in $2.5 \%$ potassium dichromate solution at $28^{\circ} \mathrm{C}$ was observed by fluorescence microscopy. The sporulation process encompassed three times of nuclear division and two times of cytokinesis, and completed within $30 \mathrm{~h}$ (Figure 4). After the second nuclear division (Figures 4A-C), four nuclei formed and the cell body became quadrilateral (Figures 4D,E). Then four sporoblasts symmetrically formed in four directions. After the first cytokinesis, these concave grooves separated from the mother cell into four symmetric daughter cells (Figures $4 \mathrm{~F}-\mathrm{H}$ ). At the same time, oocyst residual body formed during the second nuclear division after releasing from the mother cell (Figure 4H). Two sporozoites formed in each daughter cell after the third nuclear division and the second cytokinesis (Figures 4G-L). Each sporozoite contained two refractile bodies of different sizes, which began to form before the second nuclear division and were not fluorescent under the fluorescence microscope (Figure 4I).

\section{Reproductivity and Immunogenicity of the Transgenic E. intestinalis}

First, oocyst outputs per rabbit inoculated with both strains were determined (Table 1). Oocyst yield of the transgenic E. intestinalis was significantly less than that of the wild type (by $\sim 87 \%$; $p \leq 0.05)$, indicating a weakened reproductivity of transgenic E. intestinalis.

Second, rabbits were challenged with a high dose of wild type $E$. intestinalis oocysts to determine the immunogenicity of the transgenic parasites. No case of diarrhea or mortality was seen after challenge in immunized rabbits. Although body weights of immunized rabbits were lower than that of the non-immunized non-challenged group, there was no significant difference between the two immunized groups ( $p>0.05$; Figure 5). Body weight of the non-immunized, challenged group was significantly lower than all other groups. In addition, similar oocyst reductions were detected in the wild type and transgenic parasites immunized groups after challenge (Table 1),

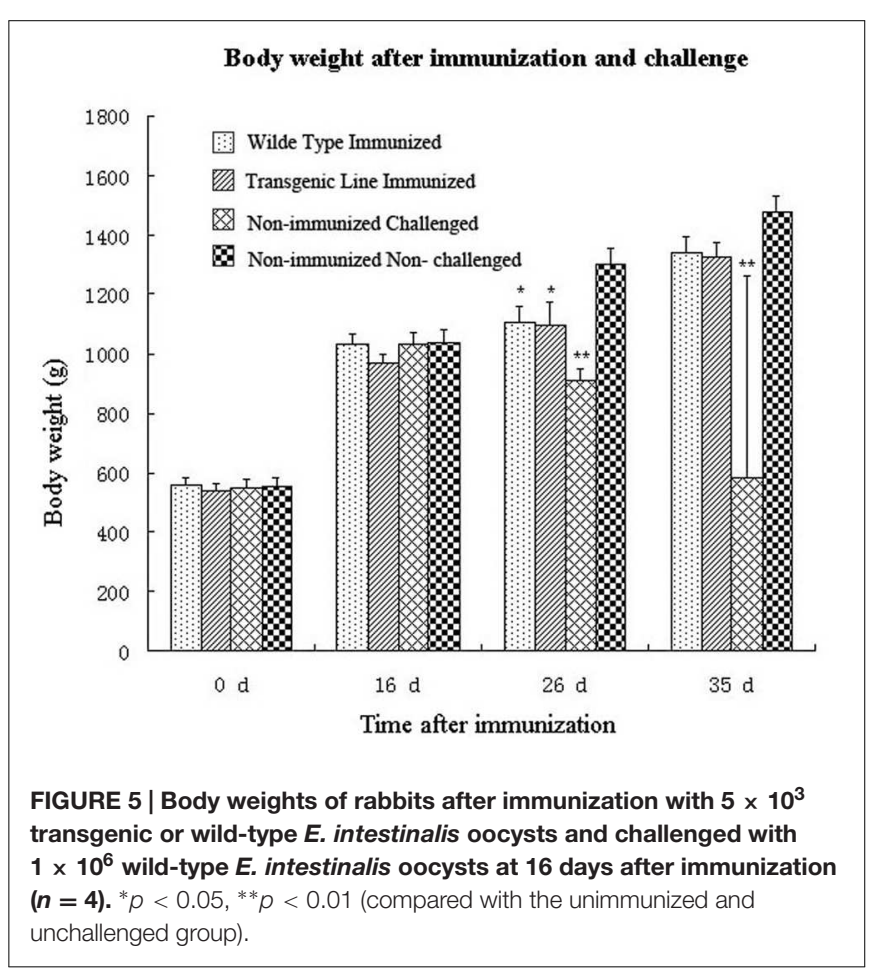


indicating that the transgenic E. intestinalis possessed similar immunogenicity and protective efficacy to the wild type parasites against homologous parasite infection.

\section{DISCUSSION}

In our study, a stable transfection system was successfully established in E. intestinalis. This is the first report on transgenesis of rabbit coccidia and an important progress in genetic manipulation of Eimerian parasites.

The plasmid used in our study contained regulatory sequences originating from E. tenella. Another study on transfection of E. mitis was conducted using similar method, as well (Qin et al., 2014). Regulatory sequences of E. tenella housekeeping genes are highly conserved and functional among Eimeria spp., which is valuable in studying other Eimeria spp. since only a few species including E. tenella have been genomically mapped (Shirley, 2000; Chapman et al., 2013; Blake, 2015). Stably transfected rabbit Eimeria spp. had not been previously achieved because it was time- consuming to rear coccidia-free rabbits, E. intestinalis sporozoites are more difficult to purify than E. tenella, and inoculation of transfected sporozoites into the small intestine of rabbits requires abdominal surgery.

In our transgenic $E$. intestinalis, the fluorescent protein was expressed mainly in the nuclei of the parasites. It is valuable for the identification of parasite in different developmental stages. In a previous study on transgenic E. mitis, a pair of flagella of microgametes was newly identified (Qin et al., 2014). In our study, apart from major features of parasite development consistent with earlier reports, we found some important features which have not been previously described. Trophozoites were detected as early as $4 \mathrm{~h}$ pi, which was considerably sooner than 24-48 h pi. reported previously (Licois et al., 1992; Yin et al., 1993). Besides lower jejunum and ileum, parasites of the

\section{REFERENCES}

Blake, D. P. (2015). Eimeria genomics: where are we now and where are we going? Vet. Parasitol. 212, 68-74. doi: 10.1016/j.vetpar.2015.05.007

Chapman, H. D., Barta, J. R., Blake, D., Gruber, A., Jenkins, M., Smith, N. C., et al. (2013). A selective review of advance in coccidiosis research. Adv. Parasitol. 83, 93-171. doi: 10.1016/B978-0-12-407705-8.00002-1

Clark, J. D., Oakes, R. D., Redhead, K., Crouch, C. F., Francis, M. J., Tomley, F. M., et al. (2012). Eimeria species parasites as novel vaccine delivery vectors: anticampylobacter jejuni protective immunity induced by Eimeria tenella-delivered CjaA. Vaccine 30, 2683-2688. doi: 10.1016/j.vaccine.2012.02.002

Coudert, P., Licois, D., and Drouet-Viard, F. (1995). "Eimeria species and strains of the rabbits," in Guidelines on Techniques in Coccidiosis Research, eds J. Eckert, R. Braun, M. W. Shirley, and P. Coudert (Brussels: European Commission), 52-73.

Coudert, P., Licois, D., Provôt, F., and Drouet-Viard, F. (1993). Eimeria sp. from rabbit (Oryctolagus cuniculus): pathogenicity and immunogenicity of Eimeria intestinalis. Parasitol. Res. 79, 186-190. doi: 10.1007/BF00 931890

de Koning-Ward, T. F., Janse, C. J., and Waters, A. P. (2000). The development of genetic tools for dissecting the biology of malaria parasites. Annu. Rev. Microbiol. 54, 157-185. doi: 10.1146/annurev.micro.54.1.157

Donald, R. G. K., and Roos, D. S. (1993). Stable molecular transformation of Toxoplasma gondii: a selectable dihydrofolate reductase-thymidylate synthase gametogony stage were also found in duodenum and vermiform appendix.

The expression level of exogenous protein is generally low in transgenic Eimeria spp. Because of the low foreign protein expression and low dose of virulent Eimeria spp. that can be safely administered as a vaccine vector, vaccination using virulent strains of Eimeria spp. would induce low exogenous protein-specific immune response. Thus, low virulent yet immunogenic strains as vaccine vectors, for instance, the transgenic E. intestinalis developed by us may be able to stimulate robust immunity without adversely affecting animal growth and meat production.

\section{AUTHOR CONTRIBUTIONS}

TS and XS designed this study. TS carried out the experiments with the help of GT, GB, JS, LH, and YF. XS supervised the study implementation. TS drafted the manuscript. GT and XS contributed the revision of the manuscript. All authors read and approved the final version of the manuscript.

\section{FUNDING}

This research was supported by the National Natural Science Foundation of China (project numbers 31101810 \& 31472182) and China Agriculture Research System (CARS-44).

\section{ACKNOWLEDGMENT}

We are most grateful to Dr. Jin Zhu (University of Canberra, Australian Capital Territory, Australia) for comments and revisions that greatly improved this manuscript.

marker based on drug-resistance mutations in malaria. Proc. Natl. Acad. Sci. U.S.A. 90, 11703-11707. doi: 10.1073/pnas.90.24.11703

Goodewardene, R., Daily, J., Kaslow, D., Sullivan, T. J., Duffy, P., Carter, R., et al. (1993). Transfection of the malaria parasite and expression of firefly luciferase. Proc. Natl. Acad. Sci. U.S.A. 90, 5234-5236. doi: 10.1073/pnas.90.11.5234

Huang, X., Zou, J., Xu, H., Ding, Y., Yin, G., Liu, X., et al. (2011). Transgenic Eimeria tenella expressing enhanced yellow fluorescent protein targeted to different cellular compartments stimulated dichotomic immune responses in chickens. J. Immunol. 187, 3595-3602. doi: 10.4049/jimmunol.1100043

Kurth, M., and Entzeroth, R. (2009). Reporter gene expression in cell culture stages and oocysts of Eimeria nieschulzi (Coccidia, Apicomplexa). Parasitol. Res. 104, 303-310. doi: 10.1007/s00436-008-1192-0

Li, M. Z., Wang, H., Liu, J., Hao, P., Ma, L., and Liu, Q. (2015a). The apoptotic role of metacaspase in Toxoplasma gondii. Front. Microbiol. 6:1560. doi: $10.3389 /$ fmicb. 2015.01560

Li, Z. R., Tang, X. M., Suo, J. X., Qin, M., Yin, G. W., Liu, X. Y., et al. (2015b). Transgenic Eimeria mitis expressing chicken interleukin 2 stimulated higher cellular immune response in chickens compared with the wild-type parasites. Front. Microbiol. 6:533. doi: 10.3389/fmicb.2015.00533

Licois, D., Coudert, P., Bahagia, S., and Rossi, G. L. (1992). Endogenous development of Eimeria intestinalis in rabbits (Oryctolagus cuniculus). J. Parasitol. 78, 1041-1048. doi: 10.2307/3283227

Licois, D., Coudert, P., Boivin, M., Drouet-Viard, F., and Provot, F. (1990). Selection and characterization of a precocious line of Eimeria 
intestinalis, an intestinal rabbit coccidium. Parasitol. Res. 76, 192-198. doi: 10.1007/BF00930814

Liu, X. Y., Shi, T. Y., Ren, H., Su, H. L., Yan, W. C., and Suo, X. (2008). Restriction enzyme mediated transfection improved transfection efficiency in vitro in Apicomplexan parasite Eimeria tenella. Mol. Biochem. Parasitol. 161, 72-75. doi: 10.1016/j.molbiopara.2008. 06.006

Pakandl, M. (2009). Coccidia of rabbit: a review. Folia Parasitol. (Praha) 56, 153-166. doi: 10.14411/fp.2009.019

Qin, M., Liu, X. Y., Tang, X. M., Suo, J. X., Tao, G. R., and Suo, X. (2014). Transfection of Eimeria mitis with yellow fluorescent protein as reporter and the endogenous development of the transgenic parasite. PLOS ONE 9:e114188. doi: 10.1371/journal.pone.0114188

Shi, T. Y., Bao, G. L., Fu, Y., Suo, X., and Hao, L. L. (2014). A low-virulence Eimeria intestinalis isolate from rabbit (Oryctolagus cuniculus) in China: molecular identification, pathogenicity, and immunogenicity. Parasitol. Res. 113, 10851090.

Shi, T. Y., Liu, X. Y., Hao, L. L., Li, J. D., Gh, A. N., Abdille, M. H., et al. (2008). Transfected Eimeria tenella could complete its endogenous development in vitro. J. Parasitol. 94, 978-980. doi: 10.1645/GE1412.1

Shirley, M. W. (2000). The genome of Eimeria spp. with special reference to Eimeria tenella- a coccidium from the chicken. Int. J. Parasitol. 30, 485-493. doi: 10.1016/S0020-7519(99)00183-6

Sibley, L. D., Messina, M., and Niesman, I. R. (1994). Stable DNA transformation in the obligate intracellular parasite Toxoplasma gondii by complementation of tryptophan auxotrophy. Proc. Natl. Acad. Sci. U.S.A. 91, 5508-5512. doi: 10.1073/pnas.91.12.5508

Soldati, D., and Boothroyd, J. C. (1993). Transient transfection and expression in the obligate intracellular parasite Toxoplasma gondii. Science 260, 349-352. doi: $10.1126 /$ science. 8469986

Wallach, M. (2010). Role of antibody in immunity and control of chicken coccidiosis. Trends Parasitol. 26, 382-387. doi: 10.1016/j.pt.2010. 04.004

Yan, W. C., Liu, X. Y., Shi, T. Y., Hao, L. L., Tomley, F. M., and Suo, X. (2009). Stable transfection of Eimeria tenella: constitutive expression of the YFP-YFP molecule throughout the life cycle. Int. J. Parasitol. 39, 109-117. doi: 10.1016/j.ijpara.2008. 06.013

Yin, P. Y., Lin, K. H., and Zhang, W. W. (1993). Studies on sporogony and merogony of Eimeria intestinalis cheissin, 1948. Acta Zool. Sin. 39, 189-198.

Conflict of Interest Statement: The authors declare that the research was conducted in the absence of any commercial or financial relationships that could be construed as a potential conflict of interest.

Copyright (C) 2016 Shi, Tao, Bao, Suo, Hao, Fu and Suo. This is an open-access article distributed under the terms of the Creative Commons Attribution License (CC BY). The use, distribution or reproduction in other forums is permitted, provided the original author(s) or licensor are credited and that the original publication in this journal is cited, in accordance with accepted academic practice. No use, distribution or reproduction is permitted which does not comply with these terms. 